\title{
Model-based Real-time Thermal Fault Diagnosis of Lithium-ion Batteries
}

\author{
Satadru Dey**. Zoleikha Abdollahi Biron*. Sagar Tatipamula*. Nabarun Das*. \\ Sara Mohon*. Beshah Ayalew*. Pierluigi Pisu*
}

\author{
** Civil and Environmental Engineering, University of California, Berkeley, CA 94720, USA \\ *Clemson University - International Center for Automotive Research, Greenville, SC 29607, USA \\ (e-mails: satadru86@ berkeley.edu, $\{$ zabdoll, statipa, nabarud, smohon, beshah, pisup\}@ clemson.edu).
}

\begin{abstract}
Ensuring safety and reliability is a critical objective of advanced Battery Management Systems (BMSs) for Li-ion batteries. In order to achieve this objective, advanced BMS must implement diagnostic algorithms that are capable of diagnosing several battery faults. One set of such critical faults in Li-ion batteries are thermal faults which can be potentially catastrophic. In this paper, a diagnostic algorithm is presented that diagnoses thermal faults in Lithium-ion batteries. The algorithm is based on a two-state thermal model describing the dynamics of the surface and the core temperature of a battery cell. The residual signals for fault detection are generated by nonlinear observers with measured surface temperature and a reconstructed core temperature feedback. Furthermore, an adaptive threshold generator is designed to suppress the effect of modeling uncertainties. The residuals are then compared with these adaptive thresholds to evaluate the occurrence of faults. Simulation and experimental studies are presented to illustrate the effectiveness of the proposed scheme.
\end{abstract}

Keywords: Lithium-ion Batteries, Fault Diagnosis, Thermal Faults, Observer, Adaptive Threshold

\section{Introduction}

Li-ion batteries are becoming the dominant energy storage solutions in electric and hybrid electric vehicle applications due to their high specific power and energy as well as relatively longer life. For such demanding applications, these batteries must perform reliably and pose no safety threats. One such issue that greatly impacts the safety, stability, performance and life of a Li-ion battery is thermal behaviour. There are many reported instances of thermal runaway in $\mathrm{Li}$ ion batteries leading to fires, for example, in Wang et al (2012). Temperature imbalances among multiple cells in a battery pack could also greatly influence the aging behaviour of the battery, as reported in Bandhauer et al (2011). Therefore, thermal management strategies are essential to mitigate these effects and avoid catastrophic failures of the battery. In line with these needs, in this paper, a model-based real time diagnostics scheme is proposed for the detection and isolation of a set of faults that affect surface and core temperature dynamics of the battery.

Numerous thermal modelling studies have been conducted on rechargeable Li-ion batteries. The objectives of the proposed thermal models fall under two categories: 1) to ensure proper thermal management system design under normal (faultless) operating conditions, and 2) to provide predictive capability for thermal abuse responses (see Doughty et al (2002)). Comprehensive thermal models developed in Kim et al (2007), Guo et al (2010), Maleki et al (2003) and Hallaj et al (1999) provide a more accurate understanding of the cell behaviour under abuse conditions like overheating and external short circuits. Since these 3D models require high computational capabilities, currently their use in battery management systems (BMS) may only be viable for industrial/stationary storage applications (see Chen et al (2005)). In vehicle applications, simple one-dimensional thermal models that compute the average lumped temperature of the cell are viable for real-time BMS implementations. For example, one such model is presented in Smith et al (2006). As a trade-off between the comprehensive and simplified modelling approaches, a two-state thermal model that predicts the surface and core temperature of a battery cell has also been proposed in Doughty et al (2002) and Park et al (2003). These two-state models provide more information than the lumped model while retaining computational simplicity. In this paper the two-state thermal model is adopted to design and analyze our proposed thermal fault diagnosis scheme.

In the literature, several estimation techniques are proposed for Li-ion batteries. Two main categories of such estimation schemes are: 1) electrochemical model-based approaches: Dey et al (2015a), Dey et al (2015b), Klein et al (2013), Moura et al (2012) and, 2) equivalent circuit model-based approaches: He et al (2011), Charkhgard et al (2010), Hu et al (2012), Kim (2006), Plett et al (2004), Gould et al. (2008). However, fewer works address the temperature estimation problem of Li-ion batteries. An adaptive observer for core temperature estimation was presented in Lin et al (2013). A battery cell temperature estimation algorithm was presented in Debert et al (2013) based on Linear Parameter Varying (LPV) thermal model and a polytopic observer. Richardson et 
al (2014) presented a method for battery internal temperature estimation by combining impedance and surface temperature measurements. A Kalman filter-based approach is presented in Kim et al (2014) to estimate the radial temperature distribution of a cylindrical battery cell under unknown cooling conditions. Papazoglou et al (2014) and Feig et al (2014) have also explored Kalman filtering techniques for battery temperature estimation. The correlation between thermal imbalance and cell degradation has been studied in Merla et al (2016).

Compared to the estimation problems, battery real-time diagnostics problems are even less explored or reported in the literature. The reported works are mainly concerned with sensor and/or actuator fault detection (see Marcicki et al (2010), Liu et al (2014), Lombardi et al (2014), Dey et al $(2015 \mathrm{c})$ ). Singh et al (2013) presented an algorithm for detecting over-charge and over-discharge faults. An electrochemical fault diagnostics scheme has been recently presented in Dey et al (2015d). Marcicki et al (2010), Liu et al (2014) and Biron et al (2015) used a one-state lumped thermal model for fault detection. However, none of these approaches exploit the two-state thermal model which can potentially capture more information on thermal dynamics including the core temperature. Therefore, these existing approaches may not be able to detect and isolate internal thermal faults. The main contribution of the present paper is that it proposes a two-state thermal model-based diagnostic scheme that takes the core temperature dynamics into account. The novelty of the proposed diagnostic scheme with respect to the existing thermal diagnostic schemes (Marcicki et al (2010) and Liu et al (2014)), lies in its capability of detecting and isolating two sets of faults: faults that affect the core temperature dynamics (e.g. thermal runaway fault) and faults that affect the surface temperature dynamics (e.g. convection fault). Note that, this work explores the problem of thermal fault diagnosis which is different from the previous works of present authors that explores electrochemical state estimation (Dey et al (2015a)), combined state and parameter estimation (Dey et al (2015b)), sensor fault diagnosis (Dey et al (2015c)) and, electrochemical fault diagnosis (Dey et al (2015d)) problems.

Model-based fault diagnosis of dynamic systems has been an active research area for several decades and explored for various applications. For some recent results in this area, please refer to Gao et al (2015). There are some existing estimation-based approaches for fault diagnosis, e.g. sliding mode observer (Edwards et al (2000)), Kalman filter (Alessandri et al (1999)), $H_{\infty}$ filter (Zhong et al (2003)). Essentially, these approaches utilize output estimation errors as residual signals, which are used for fault diagnosis. Typically, a fault is diagnosed if the residual is non-zero. However, the sliding mode observers may be ineffective in diagnosing incipient faults due to chattering problems. The $H_{\infty}$ filter also suffers from similar problem as it might mask the incipient faults. The Kalman filter based approaches generally assume a Gaussian probability distribution of the uncertainties which might not be true in practical applications. Therefore, a Lyapunov-based nonlinear observer approach is adopted in this work which does not suffer from the aforementioned issues.

One of the main challenges in model-based diagnostics arises from the uncertainties. Due to modelling, parametric and measurement uncertainties, the residuals will be nonzero even in no fault conditions. Several methods have been proposed to deal with fault diagnosis problem in presence of modelling uncertainties, e.g. sliding mode observers (Yan et al (2007)), adaptive estimators (Patton et al (2009), Patton et al (2010)). However, most of these approaches require assumptions on the structures of distribution matrices of the faults and uncertainties which may not always be met in practice. On the other hand, some diagnostic approaches have also been proposed to deal with modelling uncertainties without any assumptions on the distribution matrices. One of such approaches is the use of non-zero thresholds (EmamiNaeini et al (1988)). Essentially, the residuals are compared against some non-zero thresholds to conclude the occurrence of the faults. There are two types of thresholds that are used in literature, fixed thresholds (e.g. Emami-Naeini et al (1988)) and adaptive thresholds (e.g. Zhang et al (2002) in time domain, Ding et al (1991) in frequency domain). In case of dynamic uncertainties, fixed thresholds may need to be set at much higher levels therefore potentially leading to higher miss detection rates whereas lowering fixed thresholds might increase the false alarm rate. Further, fixed thresholds might not be effective for some systems where the operating conditions, control signals and uncertainties have significant variations. In such cases, adaptive thresholds might be effective in improving the robustness of the diagnostic scheme. Essentially, the adaptive threshold generator generates a dynamic threshold based on the nominal system dynamics and known bounds of the uncertainties. For implementation purpose, these approaches utilize filters that provide a dynamic threshold with which the observer output error (residual signal) is compared to diagnose the faults. In this paper, we adopt the adaptive threshold approach in conjunction with the observers to design the diagnostic scheme.

In this paper, the preliminary work on model-based thermal fault diagnosis that is presented in the brief conference paper (Dey et al (2015e)) is extended by: 1) considering a more comprehensive battery cell model including essential nonlinearities and incorporating a nonlinear observer design approach to deal with the nonlinearities, 2) considering temperature dependency of the internal resistance of the battery cell, 3) considering the effect of modelling uncertainties and designing an adaptive threshold generator to suppress their effects in the diagnostic scheme, 4) including some experimental results. An observer-based approach for model-based diagnosis is adopted here (Gertler (1998)). A nonlinear observer is designed using a two-state thermal model and voltage and temperature measurements. The output errors of the observer are used as the primary residuals, which have the idealized property of being zero in a non-faulty condition and non-zero otherwise. However, due 
to the presence of modelling uncertainties, the residuals are generally non-zero even in the absence of the faults. To deal with this issue, an adaptive threshold generator is used. The adaptive threshold generator is essentially a filter that is designed based on the nominal error dynamics of the observer and known uncertainty bounds (Zhang et al. (2002)). The adaptive threshold generator provides dynamic thresholds to which the residuals are compared. If the residual crosses the threshold a fault is declared. Simulation and experimental studies are provided to validate the proposed algorithm.

The rest of the paper is organized as follows. Section 2 describes the thermal model used for this study. Section 3 introduces the diagnostic problem and explains the fault diagnosis scheme. Section 4 presents simulation and experimental studies and Section 5 concludes the paper.

\section{Thermal Modelling of Lithium-ion Battery}

A radial thermal model (see Doughty et al (2002)) is chosen for the cylindrical Li-ion battery cell as depicted in Figure 1. It models a heat source at the core of the battery and two temperature states (the core temperature $T_{c}$ and the surface temperature $T_{s}$ ) given as

$$
\begin{aligned}
& C_{c} \dot{T}_{c}=\frac{T_{s}-T_{c}}{R_{c}}+I^{2} R \\
& C_{s} \dot{T}_{s}=-\frac{T_{s}-T_{c}}{R_{c}}+\frac{T_{f}-T_{s}}{R_{u}}
\end{aligned}
$$

where $I$ is the current, $R$ is the internal (electrical) resistance of the battery, $R_{c}$ is the thermal resistance between the battery core and battery surface, $R_{u}$ is the convective resistance between the battery surface and the surrounding air, $C_{c}$ is the heat capacity of internal battery material, $C_{s}$ is the heat capacity of the battery material at the surface, and $T_{f}$ is the surrounding air temperature.

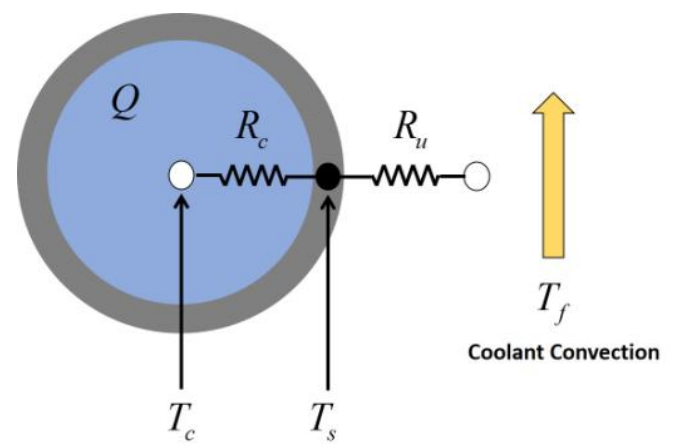

Figure 1: Battery lumped thermal model

The battery electrical behaviour is included in a zero-order equivalent electrical circuit model with open circuit voltage $E_{0}$ and internal resistance $R$ connected in series. From Kirchhoff's law the electrical equation is given by
$V=E_{0}-I R$

where $V$ is the battery's terminal voltage. Likewise, the state of charge $(S O C)$ dynamics of the battery is defined as

$$
S \dot{O} C=-I / Q
$$

where $Q$ is the battery capacity. For this paper, the $E_{0}$ is modelled as a function of $S O C$ as

$$
E_{0}=\alpha_{0}+\alpha_{1} S O C+\alpha_{2} S O C^{2}
$$

where $\alpha_{0}, \alpha_{1}$ and $\alpha_{2}$ are constant parameters that can be determined by offline identification techniques. Further, the internal resistance of the battery is modelled as a linearly parameterized function of $S O C$ and core temperature $T_{C}$.

$$
R=\beta_{0}+\beta_{1} S O C+\beta_{2} T_{C}
$$

where $\beta_{0}, \beta_{1}$ and $\beta_{2}$ are constant parameters that can be determined a priori by offline identification techniques or via online estimation schemes (see Lin et al (2013)). The experimental set up for the identification will be detailed in Section 4.1.

Remark 1: Note that, linear dependencies of $R$ on SOC and $T_{c}$ are assumed to simplify the observer design. However, the proposed framework may also be extended for nonlinear dependencies.

Sources of Uncertainties: Note that, in practice there are several sources of uncertainties that affect the performance of the proposed diagnostic scheme as mentioned below:

Modelling uncertainties: The main source of modelling uncertainty arises from the heat generation model, which does not include the effect of different electrochemical reactions (Bandhauer et al (2011)).

Measurement uncertainties: This includes measurement noises from the measured variables: voltage, current and surface temperature.

Parametric uncertainties: The main parametric uncertainty arises from battery aging (Bandhauer et al (2011)). The internal resistance $(R)$ and the battery capacity $(Q)$ are the two main parameters that change with battery age. Typically, the capacity decreases and the resistance increases. Furthermore, the thermal parameters $\left(C_{c}\right.$ and $\left.C_{s}\right)$ may also deviate due to aging. These aging effects are very difficult to capture in the nominal model.

\section{Fault Diagnosis Scheme}

Based on the nominal thermal dynamics (1)-(2), a faulty model of the battery is defined as

$$
\begin{aligned}
& C_{c} \dot{T}_{c}=\frac{T_{s}-T_{c}}{R_{c}}+I^{2} R+f_{1} \\
& C_{s} \dot{T}_{s}=-\frac{T_{s}-T_{c}}{R_{c}}+\frac{T_{f}-T_{s}}{R_{u}}+f_{2}
\end{aligned}
$$


where $f_{1}$ and $f_{2}$ represent the faults in the core temperature dynamics and surface temperature dynamics, respectively. We consider three different types of physical faults in this study.

Fault 1: Convective cooling resistance fault, which is represented by a significant deviation in the parameter $R_{u}$ from its nominal value.

Fault 2: Internal thermal resistance fault, which is modelled by change in the parameter $R_{c}$ from its nominal value.

Fault 3: Heat generation fault, which is modelled by an additional heat-generation term that contributes to the core temperature rise in the battery. Note that, when the additive heat generation fault is of high amplitude with abrupt change then it captures the effect of thermal runaway.

The mapping from these physical faults to $f_{1}$ and $f_{2}$ is given in Table I. This mapping and the additive description of the faults in (7) and (8) will be useful for designing the diagnostic scheme. Essentially, we concentrate on detecting, isolating and estimating $f_{1}$ and $f_{2}$ which is equivalent to detecting and isolating Faults 1, 2 and 3 via Table I.

The diagnostic scheme is shown in Fig. 2.

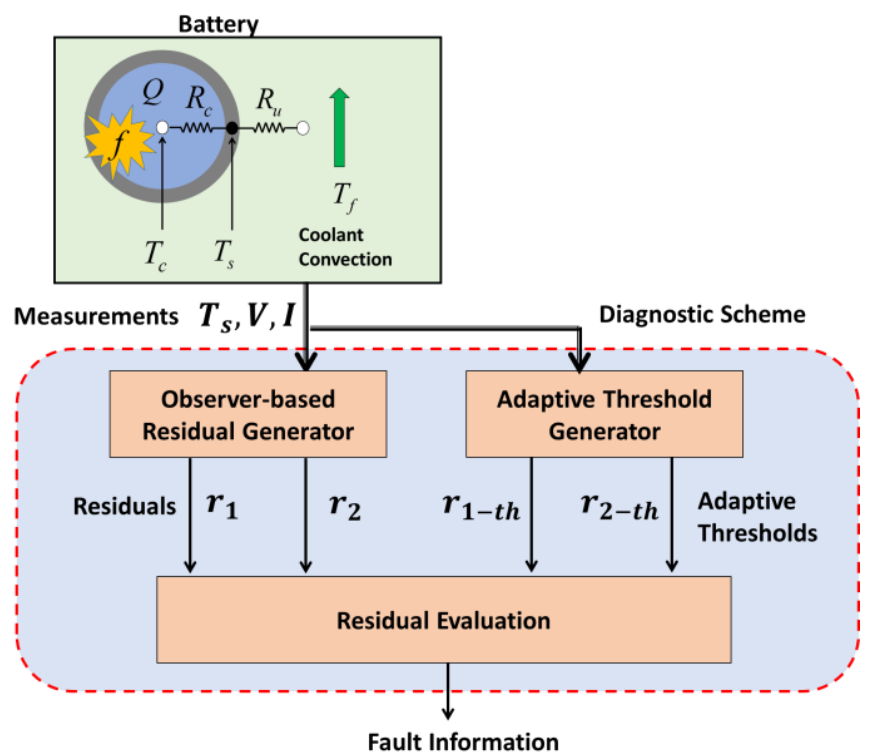

Figure 2: Diagnostic scheme

Table I: Fault mapping

\begin{tabular}{cc}
\hline Actual Faults & Fault Map \\
\hline Fault 1 & $f_{1}=0, f_{2} \neq 0$ \\
\hline Fault 2 & $f_{1} \neq 0, f_{2} \neq 0$ \\
\hline Fault 3 & $f_{1} \neq 0, f_{2}=0$ \\
\hline
\end{tabular}

\subsection{Diagnostic Scheme}

Considering the nominal battery model in (1)-(2), the statespace representation can be written as

$$
\begin{aligned}
& \dot{x_{1}}=a_{11} x_{1}+a_{12} x_{2}+b_{11} u_{1}{ }^{2} f_{R}\left(S O C, x_{1}\right) \\
& \dot{x_{2}}=a_{21} x_{1}+a_{22} x_{2}+b_{22} u_{2}
\end{aligned}
$$

where $x_{1}=T_{c}, \quad x_{2}=T_{s}, \quad u_{1}=I, \quad u_{2}=T_{f}, \quad a_{11}=\frac{-1}{R_{c} C_{c}}$, $a_{12}=\frac{1}{R_{c} C_{c}}, \quad a_{21}=\frac{1}{R_{c} C_{s}}, \quad a_{22}=\frac{-1}{R_{u} C_{s}}-\frac{1}{R_{c} C_{s}}, \quad b_{11}=\frac{1}{C_{c}}, b_{22}=$ $\frac{1}{R_{u} C_{s}}, f_{R}\left(S O C, x_{1}\right)=R=\beta_{0}+\beta_{1} S O C+\beta_{2} x_{1}$.

Now, using the terminal voltage expression (3), open circuit voltage expression (5) and internal resistance expression (6), the internal temperature can be written as

$R=\frac{E_{0}-V}{I}$

$\Rightarrow \beta_{0}+\beta_{1} S O C+\beta_{2} T_{c}=\frac{\alpha_{0}+\alpha_{1} S O C+\alpha_{2} S O C^{2}-V}{I}$

$\Rightarrow T_{c}$

$=\frac{\frac{\alpha_{0}+\alpha_{1} S O C+\alpha_{2} S O C^{2}-V}{I}-\left(\beta_{0}+\beta_{1} S O C\right)}{\beta_{2}}$

With the available measurements of current and voltage, and assuming that the SOC is available via Coulomb counting (with available accurate enough current measurement) using (4), the internal temperature can be reconstructed as above and used as an output of the system. Therefore, the following outputs are defined

$y_{1}=x_{1}$

$y_{2}=x_{2}$

where $y_{1}$ is the reconstructed core temperature $\left(T_{c}\right)$ and $y_{2}$ is the measured surface temperature $\left(T_{S}\right)$.

Remark 2: In batteries, there is no direct measurement of the core temperature $\left(T_{c}\right)$. However, to isolate the faults $f_{1}$ and $f_{2}$, core temperature information is required in addition to the surface temperature $\left(T_{S}\right)$. To resolve this issue, the physical dependence of the internal resistance $(R)$ on the core temperature $\left(T_{c}\right)$ is utilized to reconstruct internal core temperature information from the measured quantities.

Remark 3: Note that at the input operating point $u_{1}{ }^{*}=0$, the internal resistance information will not show up in the terminal voltage and hence $T_{c}$ cannot be reconstructed. Essentially, one set of information from the plant is lost. Therefore, the scheme cannot isolate different faults at this operating point. However, the detection capability of the scheme remains intact. 


\section{Observer Design for Primary Residual Generation}

A nonlinear observer is designed to generate the primary residuals. Based on the plant model and measurements (9)(13), the observer structure is given by

$\dot{\hat{x}}_{1}=a_{11} \hat{x}_{1}+a_{12} \hat{x}_{2}+b_{11} u_{1}^{2} f_{R}\left(S O C, \hat{x}_{1}\right)+$

$L_{11} \tilde{y}_{1}+L_{12} \tilde{y}_{2}$

$\dot{\hat{x}}_{2}=a_{21} \hat{x}_{1}+a_{22} \hat{x}_{2}+b_{22} u_{2}+L_{21} \tilde{y}_{1}+L_{22} \tilde{y}_{2}$

$\tilde{y}_{1}=\tilde{x}_{1}$

$\tilde{y}_{2}=\tilde{x}_{2}$

where $\tilde{x}_{1}=x_{1}-\hat{x}_{1}, \tilde{x}_{2}=x_{2}-\hat{x}_{2}$ and SOC is determined via coulomb counting. Next, we state the main result of the observer design.

Main result: Consider the observer dynamics (14)-(17) and the plant model (9)-(13). Furthermore, consider that the observer gains satisfy the following conditions: $L_{12}=a_{12}$, $L_{21}=a_{21}, L_{22}>0$ and $L_{11}>b_{11} \beta_{2} u_{1}{ }^{2}$ for all admissible values of $u_{1}$. Then the observer error $\tilde{x}_{1}$ and $\tilde{x}_{2}$ will asymptotically converge to zero as $t \rightarrow \infty$ under no fault condition (i.e. $f_{1}, f_{2}=0$ ). Under faulty condition, (i.e. $f_{1}, f_{2} \neq 0$ ), the observer error $\tilde{x}_{1}$ and $\tilde{x}_{2}$ will be bounded to some non-zero values depending on the size of the faults and the observer gains.

Next, we will analyse the observer error dynamics under no fault and faulty conditions via Lyapunov's direct method.

Convergence analysis of the observer error under no fault condition: Now, under nominal condition (no fault case, i.e. $\left.f_{1}, f_{2}=0\right)$, subtracting (14)-(17) from (9)-(13), the dynamics of the observer error is

$\dot{\tilde{x}}_{1}=a_{11} \tilde{x}_{1}+a_{12} \tilde{x}_{2}+b_{11} \beta_{2} u_{1}{ }^{2} \tilde{x}_{1}-L_{11} \tilde{y}_{1}-L_{12} \tilde{y}_{2}$

$\dot{\tilde{x}}_{2}=a_{21} \tilde{x}_{1}+a_{22} \tilde{x}_{2}-L_{21} \tilde{y}_{1}-L_{22} \tilde{y}_{2}$

To analyze the convergence of the observer error, consider the following Lyapunov function candidate

$V=\frac{1}{2}\left(\tilde{x}_{1}^{2}+\tilde{x}_{2}{ }^{2}\right)$

Therefore, the derivative of the Lyapunov function candidate can be written as

$\dot{V}=\tilde{x}_{1} \dot{\tilde{x}}_{1}+\tilde{x}_{2} \dot{\tilde{x}}_{2}$

$=a_{11} \tilde{x}_{1}{ }^{2}+a_{12} \tilde{x}_{1} \tilde{x}_{2}+b_{11} \beta_{2} u_{1}{ }^{2} \tilde{x}_{1}{ }^{2}-L_{11} \tilde{x}_{1}{ }^{2}-L_{12} \tilde{x}_{1} \tilde{x}_{2}$

$+a_{21} \tilde{x}_{1} \tilde{x}_{2}+a_{22} \tilde{x}_{2}{ }^{2}-L_{21} \tilde{x}_{1} \tilde{x}_{2}-L_{22} \tilde{x}_{2}{ }^{2}$

Next, we determine the conditions on the observer gains for which we have $\dot{V}<0$ based on the above equation. Note that, $\dot{V}<0$ implies the asymptotic convergence of the observer error $\tilde{x}_{1}$ and $\tilde{x}_{2}$ to zero as $t \rightarrow \infty$. With the choice of $L_{12}=$ $a_{12}$ and $L_{21}=a_{21}, \dot{V}$ becomes
$\dot{V}=\left\{a_{11}+b_{11} \beta_{2} u_{1}{ }^{2}-L_{11}\right\} \tilde{x}_{1}{ }^{2}+\left\{a_{22}-L_{22}\right\} \tilde{x}_{2}{ }^{2}$

Note that $a_{11}, a_{22}<0$ from physical properties of the battery. Now, choosing $L_{22}>0$ and $L_{11}$ such that $L_{11}>b_{11} \beta_{2} u_{1}{ }^{2}$ for all admissible values of $u_{1}$, the following can be written

$\dot{V}=-m_{1} \tilde{x}_{1}{ }^{2}-m_{2} \tilde{x}_{2}{ }^{2}$

where $m_{1}=-\left\{a_{11}+b_{11} \beta_{2} u_{1}^{2}-L_{11}\right\}>0$ and $m_{2}=$ $-\left\{a_{22}-L_{22}\right\}>0$. For design, $L_{11}=m_{1}+a_{11}+b_{11} \beta_{2} u_{1}^{2}$ can be chosen where $m_{1}>0$ is a constant design parameter of the user's choice. Therefore, $\dot{V}<0$ and the state estimation error $\tilde{x}_{1}$ and $\tilde{x}_{2}$ achieve asymptotic convergence to zero as $t \rightarrow \infty$ under no fault. Note that, high values of $L_{11}$ and $L_{22}$ will improve the convergence time, but also amplify the measurement noise at the same time. Therefore, the choice of $L_{11}$ (or $m_{1}$ ) and $L_{22}$ should be made keeping this trade-off in mind.

Remark 4: As discussed before, the core temperature cannot be reconstructed from the terminal voltage measurement at the operating point $u_{1}{ }^{*}=0$. Therefore, the output error injection must be made zero by choosing $L_{11}{ }^{*}, L_{21}{ }^{*}=0$ only at that operating point (or practically, in its vicinity).

Convergence analysis of the observer error under faults: Now, under faults (i.e. $f_{1}, f_{2} \neq 0$ ), subtracting (14)-(17) from (9)-(13), the dynamics of the observer error is

$\dot{\tilde{x}}_{1}=a_{11} \tilde{x}_{1}+a_{12} \tilde{x}_{2}+b_{11} u_{1}{ }^{2}\left\{\beta_{2} \tilde{x}_{1}\right\}+f_{1}-L_{11} \tilde{y}_{1}-$ $L_{12} \tilde{y}_{2}$

$\dot{\tilde{x}}_{2}=a_{21} \tilde{x}_{1}+a_{22} \tilde{x}_{2}+b_{22} u_{2}+f_{2}-L_{21} \tilde{y}_{1}-L_{22} \tilde{y}_{2}$

To analyze the convergence of the observer error under faults, we choose the same Lyapunov function candidate and same observer gains as the no fault case. Then, the derivative of the Lyapunov function candidate can be written as

$\dot{V}=-m_{1} \tilde{x}_{1}{ }^{2}-m_{2} \tilde{x}_{2}{ }^{2}+\tilde{x}_{1} f_{1}+\tilde{x}_{2} f_{2}$

Using the bounding argument $a b \leq|a||b|$, (25) can be bounded as

$\dot{V} \leq-m_{1}\left|\tilde{x}_{1}\right|^{2}-m_{2}\left|\tilde{x}_{2}\right|^{2}+\left|\tilde{x}_{1}\right|\left|f_{1}\right|+\left|\tilde{x}_{2}\right|\left|f_{2}\right|$

$\leq-\left\{m_{1}\left|\tilde{x}_{1}\right|-\left|f_{1}\right|\right\}\left|\tilde{x}_{1}\right|-\left\{m_{2}\left|\tilde{x}_{2}\right|-\left|f_{2}\right|\right\}\left|\tilde{x}_{2}\right|$

Note that $V$ will decrease as long as the following conditions are satisfied: $\left|\tilde{x}_{1}\right|>\frac{\left|f_{1}\right|}{\left|m_{1}\right|}$ and $\left|\tilde{x}_{2}\right|>\frac{\left|f_{2}\right|}{\left|m_{2}\right|}$. However, under the conditions $\left|\tilde{x}_{1}\right| \leq \frac{\left|f_{1}\right|}{\left|m_{1}\right|}$ and $\left|\tilde{x}_{2}\right| \leq \frac{\left|f_{2}\right|}{\left|m_{2}\right|}, \dot{V}$ eventually becomes positive and $V$ does not decrease anymore. Therefore, it can be concluded that $V$ will stay in the error space in some ball of finite radius. Consequently, the state estimation errors remain bounded within a ball of radius determined by $\frac{\left|f_{1}\right|}{\left|m_{1}\right|}$ and $\frac{\left|f_{2}\right|}{\left|m_{2}\right|}$. Therefore, it can be concluded that: 
$f_{1} \neq 0=>\tilde{y}_{1} \neq 0$ and $f_{2} \neq 0=>\tilde{y}_{2} \neq 0$

Based on the above discussion, the residuals are chosen as: $r_{1}=\tilde{y}_{1}$ and $r_{2}=\tilde{y}_{2}$ along with the following fault signature table for the diagnostic scheme.

Table II: Fault signature table (' 1 ' represents nonzero and ' 0 ' represents zero)

\begin{tabular}{ccc}
\hline Fault & \multicolumn{2}{c}{ Residuals } \\
& $r_{1}$ & $r_{2}$ \\
\hline$f_{1}$ & 1 & 0 \\
\hline$f_{2}$ & 0 & 1 \\
\hline
\end{tabular}

Note: The effect of the measurement noise can be minimized by the choice of the observer gains $L_{11}$ and $L_{22}$. Lower values of these gains would result in slower observer dynamics which in turn attenuates the high frequency measurement noise. However, for a detailed design of observer gains with specific objective of attenuation of measurement noise, one may refer to Gao et al (2006).

In the next section, we will discuss how to minimize the effect of the modelling and parametric uncertainties.

\section{Adaptive Threshold Design for Residual Evaluation}

In the previous analysis, the residuals $\tilde{y}_{1}$ and $\tilde{y}_{2}$ are shown to be zero in the absence of faults. However, due to modelling, parametric and measurement uncertainties as discussed above, the residuals will be nonzero even in no fault conditions. In this paper, the adaptive threshold approach outlined in Zhang et al (2002) is adopted. Essentially, the residual signals are compared to these adaptive thresholds to diagnose the faults. To start with, the thermal model with additive uncertainties can be written as

$$
\begin{aligned}
& \dot{x}_{1}=a_{11} x_{1}+a_{12} x_{2}+b_{11} u_{1}{ }^{2} f_{R}\left(S O C, x_{1}\right)+n_{1} \\
& \dot{x}_{2}=a_{21} x_{1}+a_{22} x_{2}+b_{22} u_{2}+n_{2}
\end{aligned}
$$

where $n_{1}$ and $n_{2}$ are the model uncertainties (in the absence of faults). Note that, the uncertainties can be input and/or state dependent and treated here as unknown exogenous inputs. In the following discussion, the following form of the uncertainties is assumed

$$
\begin{aligned}
& n_{1}=k_{11} x_{1}+k_{12} x_{2}+k_{13} u_{1}+k_{14} \\
& n_{2}=k_{21} x_{1}+k_{22} x_{2}+k_{23} u_{2}+k_{24}
\end{aligned}
$$

where, $k_{i j}$ are unknown coefficients whose bounds are known, $\quad\left|k_{i j}\right| \leq k_{i j-\max }$. Note that a nonlinear parameterization is also possible for the uncertainty expressions. However, for the convenience of design, the above form is chosen where the errors of this approximation and linearization are represented by $k_{14}$ and $k_{24}$.
Remark 5: The uncertainty expressions in (28) and (29) are derived considering the modelling and parametric uncertainties in the nonlinear model (1)-(2). The main sources of uncertainties are: deviation of thermal parameters $\left(C_{s}\right.$ and $\left.C_{c}\right)$ and electrical parameters $(R$ and $Q)$; uncertainties in the ambient temperature $\left(T_{f}\right)$; other unmodeled dynamics. The nominal state-space model along with these uncertainties can be written as:

$$
\begin{aligned}
& \left(C_{c}+\Delta C_{c}\right) \dot{T}_{c}=\frac{T_{s}-T_{c}}{R_{c}}+I^{2} f_{R}\left(S O C+\Delta S O C, T_{c}\right)+\Delta_{1} \\
& \left(C_{s}+\Delta C_{s}\right) \dot{T}_{s}=-\frac{T_{s}-T_{c}}{R_{c}}+\frac{\left(T_{f}+\Delta T_{f}\right)-T_{s}}{R_{u}}+\Delta_{2}
\end{aligned}
$$

where $\Delta C_{s}, \Delta T_{f}, \Delta C_{c}$ and $\triangle S O C$ are uncertainties and $\Delta_{1}$ and $\Delta_{2}$ are the unmodelled dynamics. Comparing (30)-(31) with (28)-(29), it can be seen that the uncertainty in $C_{s}$ is included in $k_{21}, k_{22}$, and $k_{23}$; uncertainties in $C_{c}$ is included in $k_{11}$, $k_{12}$ and $k_{13}$; uncertainties in $R$ and $S O C$ (due to uncertainty in battery capacity $Q$ ) is included in $k_{13}$; uncertainties in $T_{f}$ is included in $k_{23}$. Furthermore, unmodelled dynamics are captured in $k_{14}$ and $k_{24}$.

Remark 6: The terms $k_{13} u_{1}$ and $k_{23} u_{2}$ include the effects of disturbances in input current and ambient temperature. Furthermore, the terms $k_{14}$ and $k_{24}$ include the effects of exogenous input disturbances in the system. Therefore, the effect of input disturbances is taken into account via the expressions of uncertainties in (28) and (29). In the following, the details of how to decouple the effect of such uncertainties and hence the input disturbances from the faults are shown. However, active attenuation of input disturbances is not considered as a design goal in this approach. For active attenuation of input disturbances, one may refer to Gao et al (2006).

Subtracting the observer (14)-(17) from the plant model (26)(27), the observer error dynamics in the presence of uncertainties and under no-fault condition (i.e. $f_{1}, f_{2}=0$ ), can be written as

$\dot{\tilde{x}}_{1}=-m_{1} \tilde{x}_{1}+n_{1}$

$\dot{\tilde{x}}_{2}=-m_{2} \tilde{x}_{2}+n_{2}$

with $m_{1}$ and $m_{2}$ defined after (22). Considering $n_{1}$ and $n_{2}$ as inputs to the error dynamic system (32)-(33), the solution of the state-space equation (32)-(33) can be written as:

$\tilde{x}_{1}(t)=\tilde{x}_{1}(0) e^{-m_{1} t}+\int_{0}^{t} e^{-m_{1}(t-\tau)} n_{1}(\tau) d \tau$
$\tilde{x}_{2}(t)=\tilde{x}_{2}(0) e^{-m_{2} t}+\int_{0}^{t} e^{-m_{2}(t-\tau)} n_{2}(\tau) d \tau$

Next, using the bounding argument $a b \leq|a b| \leq|a||b|$ on the product terms inside the integrals of (34)-(35), we can write

$\tilde{x}_{1}(t) \leq \tilde{x}_{1}(0) e^{-m_{1} t}+\int_{0}^{t}\left|e^{-m_{1}(t-\tau)}\right|\left|n_{1}(\tau)\right| d \tau$ 
$\tilde{x}_{2}(t) \leq \tilde{x}_{2}(0) e^{-m_{2} t}+\int_{0}^{t}\left|e^{-m_{2}(t-\tau)}\right|\left|n_{2}(\tau)\right| d \tau$

Note that, the term $e^{-m_{1} t}>0 \forall t>0$. Hence, we can write that $\left|e^{-m_{1} t}\right|=e^{-m_{1} t}$. Therefore, (36)-(37) can be re-written as

$$
\begin{aligned}
& \tilde{x}_{1}(t) \leq \tilde{x}_{1}(0) e^{-m_{1} t}+\int_{0}^{t} e^{-m_{1}(t-\tau)}\left|n_{1}(\tau)\right| d \tau \\
& \tilde{x}_{2}(t) \leq \tilde{x}_{2}(0) e^{-m_{2} t}+\int_{0}^{t} e^{-m_{2}(t-\tau)}\left|n_{2}(\tau)\right| d \tau
\end{aligned}
$$

Next, using the bounding argument $|a b| \leq|a||b|$, the bounds of the uncertain terms can be written as

$$
\begin{aligned}
& \left|n_{1}\right| \leq\left|k_{11}\right|\left|x_{1}\right|+\left|k_{12}\right|\left|x_{2}\right|+\left|k_{13}\right|\left|u_{1}\right|+\left|k_{14}\right| \\
& \left|n_{2}\right| \leq\left|k_{21}\right|\left|x_{1}\right|+\left|k_{22}\right|\left|x_{2}\right|+\left|k_{23}\right|\left|u_{1}\right|+\left|k_{24}\right|
\end{aligned}
$$

Furthermore, using the coefficient bounds $\left|k_{i j}\right| \leq k_{i j-\max }$, (40)-(41) can be written as

$$
\begin{aligned}
& \left|n_{1}\right| \\
& \leq k_{11-\max }\left|x_{1}\right|+k_{12-\text { max }}\left|x_{2}\right|+k_{13-\text { max }}\left|u_{1}\right| \\
& +k_{14-\text { max }} \\
& \left|n_{2}\right| \\
& \leq k_{21-\text { max }}\left|x_{1}\right|+k_{22-\text { max }}\left|x_{2}\right|+k_{23-\text { max }}\left|u_{1}\right| \\
& +k_{24-\text { max }}
\end{aligned}
$$

Next, applying the bound (42) into (38), we can write

$\tilde{x}_{1}(t) \leq r_{1-t h}(t)=\varepsilon_{10}+$

$\int_{0}^{t} e^{-m_{1}(t-\tau)}\left\{k_{11-\max }\left|x_{1}(\tau)\right|+k_{12-\max }\left|x_{2}(\tau)\right|+\right.$

$\left.k_{13-\max }\left|u_{1}(\tau)\right|+k_{14-\max }\right\} d \tau$

where $\varepsilon_{10}=\tilde{x}_{1}(0) e^{-m_{1} t}$ and $r_{1-t h}$ is the adaptive threshold for the residual $r_{1}=\tilde{y}_{1}=\tilde{x}_{1}$. The evolution equation (44) is equivalent to the following filter dynamics for the adaptive threshold

$$
\begin{aligned}
& \dot{z}_{11}=-m_{1} z_{11}+k_{11-\max }\left|x_{1}(t)\right| \\
& +k_{12-\max }\left|x_{2}(t)\right|+k_{13-\max }\left|u_{1}(t)\right|+k_{14-\max } \\
& r_{1-t h}=\varepsilon_{10}+z_{11}
\end{aligned}
$$

Similarly, applying the bound (43) into (39), a filter equation can be derived that generates the adaptive threshold $r_{2-t h}$ for the residual $r_{2}=\tilde{y}_{2}=\tilde{x}_{2}$.

\section{Results and Discussions}

\subsection{Identification of Lithium-ion Battery Electrical-Thermal Model}

A $\mathrm{LiCoO}_{2} /$ Graphite Li-ion battery (18650 cylindrical cell) was studied with two T-type thermocouples installed on it: one on the surface and the other inserted into the battery core. The experimental setup is shown in Fig 3. The battery is discharged through a programmable DC electronic load. The power supply supplies the power to the signal conditioner circuit, which amplifies the signals received from the thermocouples to the dSPACE ControlDesk data acquisition software working with MATLAB/Simulink.

The parameters of the battery model (1)-(6) were identified using nonlinear optimization technique that fits the two-state thermal model simulated data with the experimental data. The experimental data was collected in the SOC range of 50-80\%, temperature range of $15-40^{\circ} \mathrm{C}$ and current range up to $2 \mathrm{C}$. Current profiles include constant currents, pulse discharge and ramp currents. The parameters identified are listed in Table III.

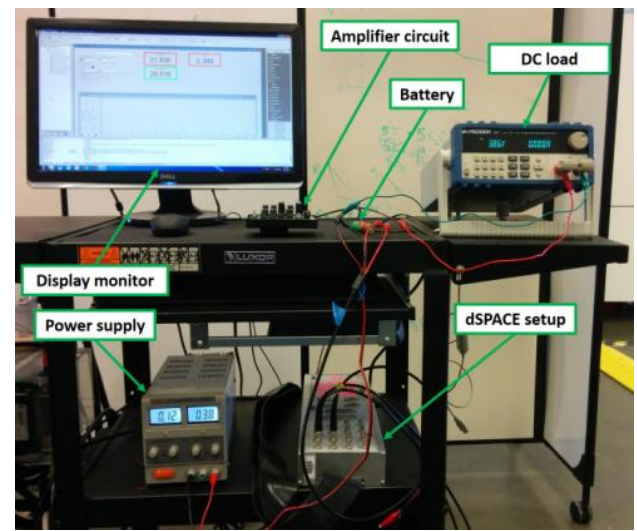

Figure 3: Experimental setup

Table III: Identified model parameters

\begin{tabular}{ccc}
\hline Parameter & Unit & Value \\
\hline$\alpha_{0}$ & $\mathrm{~V}$ & 2.28 \\
\hline$\alpha_{1}$ & $\mathrm{~V}$ & 2.2 \\
\hline$\alpha_{2}$ & $\mathrm{~V} / \mathrm{K}$ & 0.001 \\
\hline$\beta_{0}$ & $\Omega$ & 2.205 \\
\hline$\beta_{1}$ & $\Omega$ & 0.01 \\
\hline$\beta_{2}$ & $\Omega / \mathrm{K}$ & 0.007 \\
\hline$C_{c}$ & $\mathrm{~J} / \mathrm{K}$ & 18.6 \\
\hline$C_{s}$ & $\mathrm{~J} / \mathrm{K}$ & 0.1 \\
\hline$R_{c}$ & $\mathrm{~K} / \mathrm{W}$ & 150.6 \\
\hline$R_{u}$ & $\mathrm{~K} / \mathrm{W}$ & 200 \\
\hline
\end{tabular}

\subsection{Simulation Studies}

Based on the identified model parameters, the battery model is implemented in the MATLAB/Simulink platform. In this section, the simulation studies are presented to show the effectiveness of the proposed scheme. In the plant model, the uncertainties in the form (28)-(29) are injected. The coefficients are arbitrarily chosen and given in Table IV. The adaptive threshold generators are provided with the bounds of these coefficients. The current profile is a scaled and 
modified urban dynamometer drive cycle shown in Fig. 4. The corresponding temperature, voltage and residual responses are shown in Fig. 5 under no fault condition. Note that, the residual generator observers and the adaptive threshold generator filters are initialized with incorrect conditions and therefore it takes certain time for these variables to converge ( $250 \mathrm{sec}$ for residual 1 and $\sim 200 \mathrm{sec}$ for residual 2, in Fig. 5).

Table IV: Parameters of the uncertainty model in simulation

\begin{tabular}{cc}
\hline Parameter & Value \\
\hline$k_{11}$ & $10^{-3}$ \\
\hline$k_{12}$ & $2 \times 10^{-4} \sin (0.03 t)$ \\
\hline$k_{13}$ & $10^{-3}$ \\
\hline$k_{14}$ & Gaussian distribution $\left(10^{-3}, 10^{-5}\right)$ \\
\hline$k_{21}$ & $3 \times 10^{-4} \sin (0.015 t)$ \\
\hline$k_{22}$ & 0 \\
\hline$k_{23}$ & Gaussian distribution $\left(3 \times 10^{-3}, 10^{-4}\right)$ \\
\hline$k_{24}$ &
\end{tabular}

To test the effectiveness of the diagnostic scheme, three different faults have been injected in three separate cases.

Case 1: An additional heat generation term is injected at 600 sec and the corresponding residual responses are shown in Fig. 6. The nature of the fault is chosen as a ramp type representing incipient fault with ramping rate $1.25 \times 10^{-4}$ Watt/sec. Note that, this fault can be represented by $f_{1}$ in the battery's dynamics (7)-(8), and hence affects only core temperature dynamics of the battery through (7). Therefore, this particular fault, $f_{1} \neq 0$, only impacts Residual $1\left(r_{1}\right)$. As can be noted from Fig. 6, due to presence of fault, $f_{1}$, Residual $1\left(r_{1}\right)$ deviates from its nominal value and crosses the adaptive threshold after about $150 \mathrm{sec}$ of fault injection. However, fault $f_{1}$ does not show up in Residual $2\left(r_{2}\right)$, and hence this residual does not surpass its adaptive threshold.

Case 2: A fault is injected in the convective (cooling/heating) coefficient resistance $\left(R_{u}\right)$ at $500 \mathrm{sec}$. The nature of the fault is chosen as ramp type representing an incipient fault with ramping rate $0.01 \times R_{u-\text { nominal }} / \mathrm{sec}$. Note that, this fault can be represented by $f_{2}$ in the battery's dynamics (7)-(8), and hence only affects the surface temperature dynamics via (8). Therefore, this particular fault $f_{2}$, will only show up in Residual $2\left(r_{2}\right)$ according to Table. I. The residual responses corresponding to this fault are shown in Fig. 7. As can be inferred from Fig. 7, Residual $2\left(r_{2}\right)$ crosses the threshold after $t=580 \mathrm{sec}$, while Residual $1\left(r_{1}\right)$ remains between its threshold bounds.

Case 3: Another abrupt (step-like) additional heat generation fault (which can be modelled as $f_{1}$ ) is injected at $550 \mathrm{sec}$ and the corresponding residual responses are shown in Fig. 8. The amplitude of the fault is 0.025 Watt. As expected, Residual 1 $\left(r_{1}\right)$ crosses the adaptive threshold after about $590 \mathrm{sec}$ whereas Residual $2\left(r_{2}\right)$ remains within the thresholds.

Case 4: A fault is injected in the internal thermal resistance $\left(R_{c}\right)$ at $400 \mathrm{sec}$. The nature of the fault is chosen as abrupt step-like fault with amplitude $2 R_{c-n o m i n a l}$. Referring to the battery's thermal dynamics presented in (1)-(2), the internal thermal resistance $\left(R_{c}\right)$ affects both core and surface temperature dynamics. Therefore, a fault corresponding to this resistance, can be modelled as $f_{1}, f_{2} \neq 0$ in (7)-(8). Therefore, the effect of this internal thermal resistance fault will show up in Residual $1\left(r_{1}\right)$ and Residual $2\left(r_{2}\right)$. The residual responses corresponding to this fault are shown in Fig. 9. As it can be seen from Fig. 9, due to the presence of $f_{1} \neq 0$ and $f_{2} \neq 0$, Residual $1\left(r_{1}\right)$ and Residual $2\left(r_{2}\right)$ cross their thresholds after a certain time.

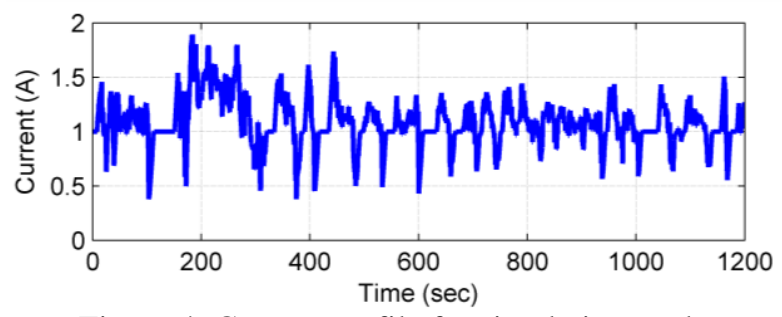

Figure 4: Current profile for simulation study
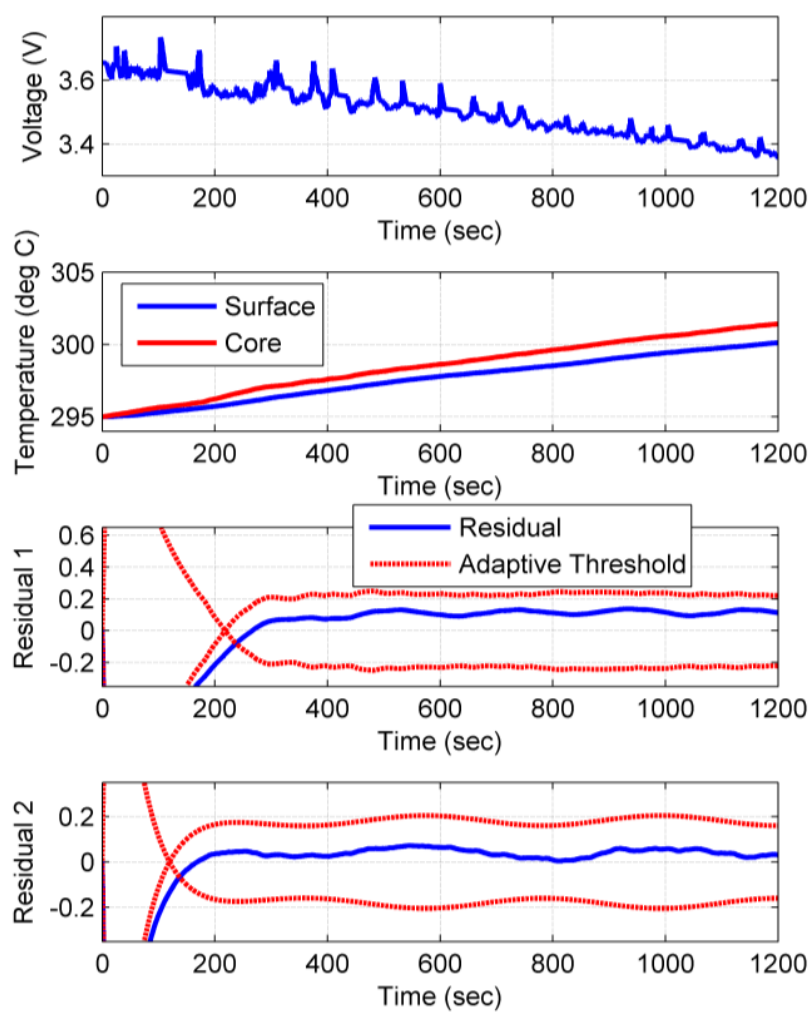

Figure 5: Voltage, temperature and residual responses under no fault condition. 

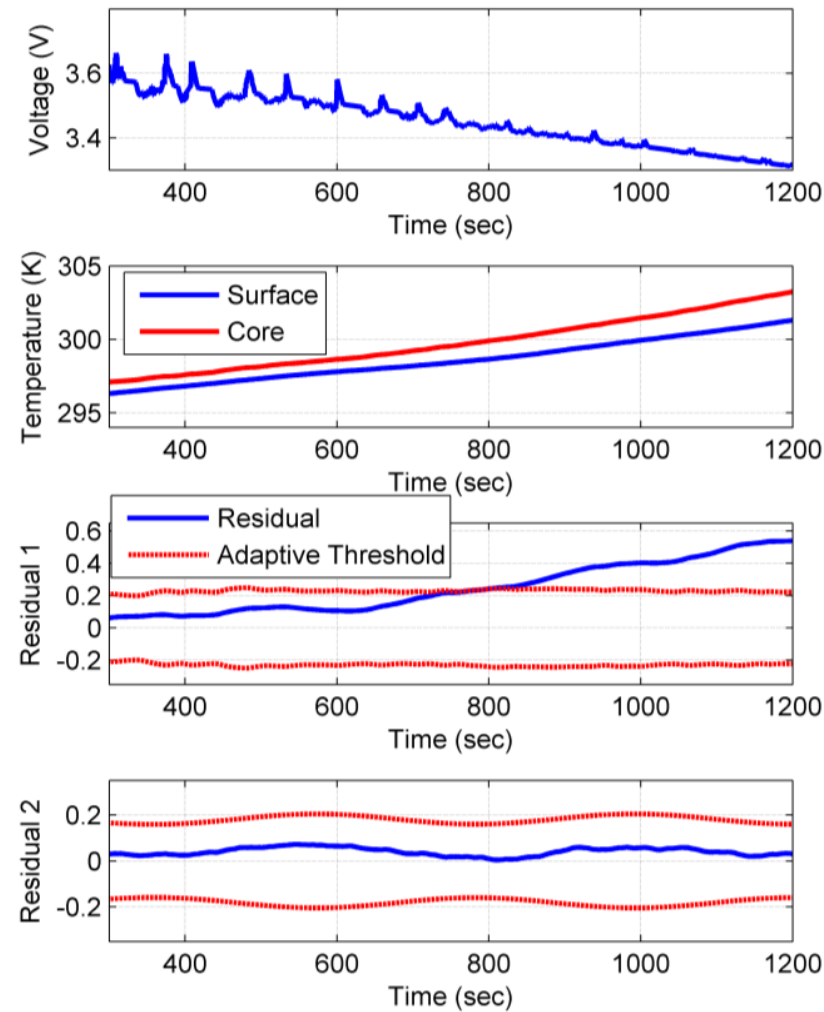

Figure 6: Residual responses under additive heat generation fault injected at $600 \mathrm{sec}$. The fault is an incipient fault with ramping rate $\mathbf{1 . 2 5} \times \mathbf{1 0}^{-4} \mathrm{Watt} / \mathrm{sec}$
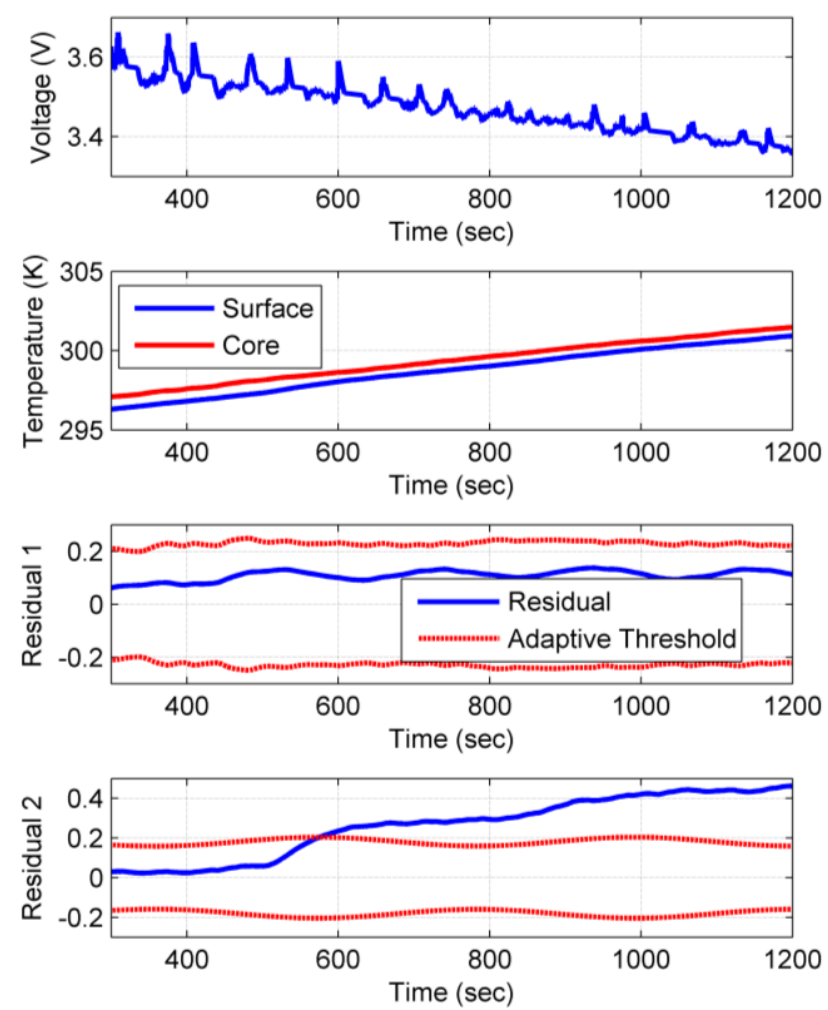

Figure 7: Residual responses under convective cooling resistance fault injected at $500 \mathrm{sec}$. The fault is a ramp-type fault with ramping rate $\mathbf{0 . 0 1} \times \mathbf{R}_{\mathbf{u}-\text { nominal }} / \mathrm{sec}$.
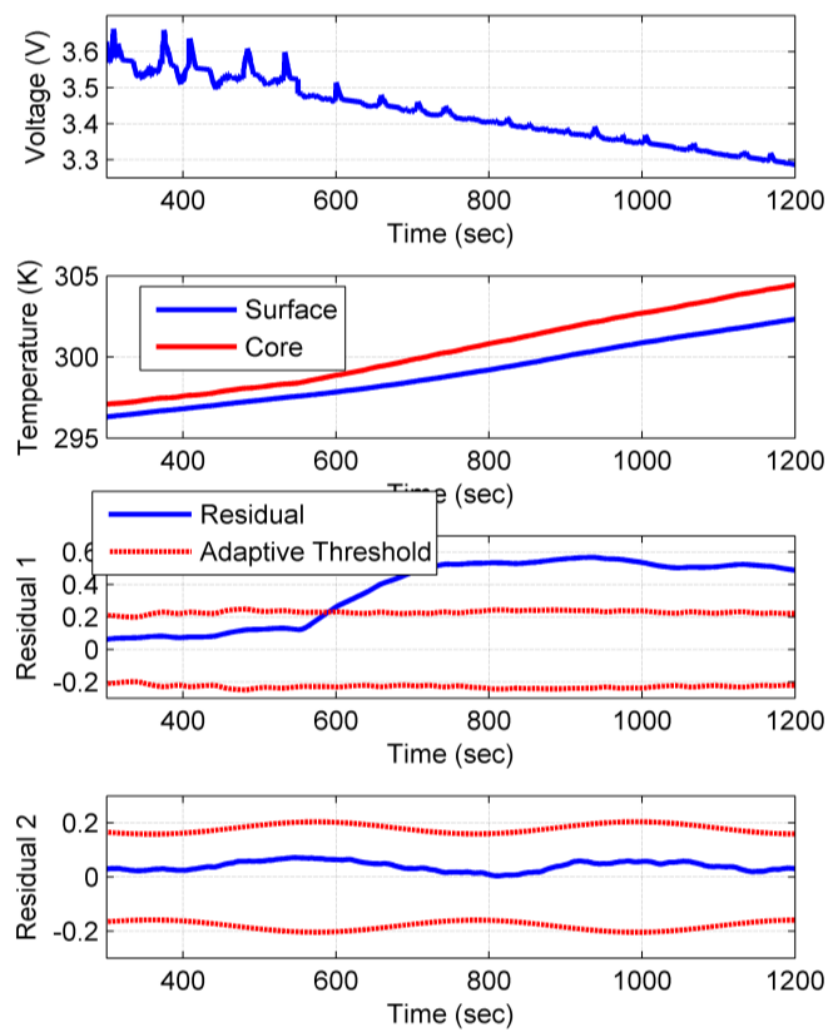

Figure 8: Residual responses under abrupt additive heat generation fault injected at $550 \mathrm{sec}$. The fault is a step-like fault with amplitude of 0.025 Watt
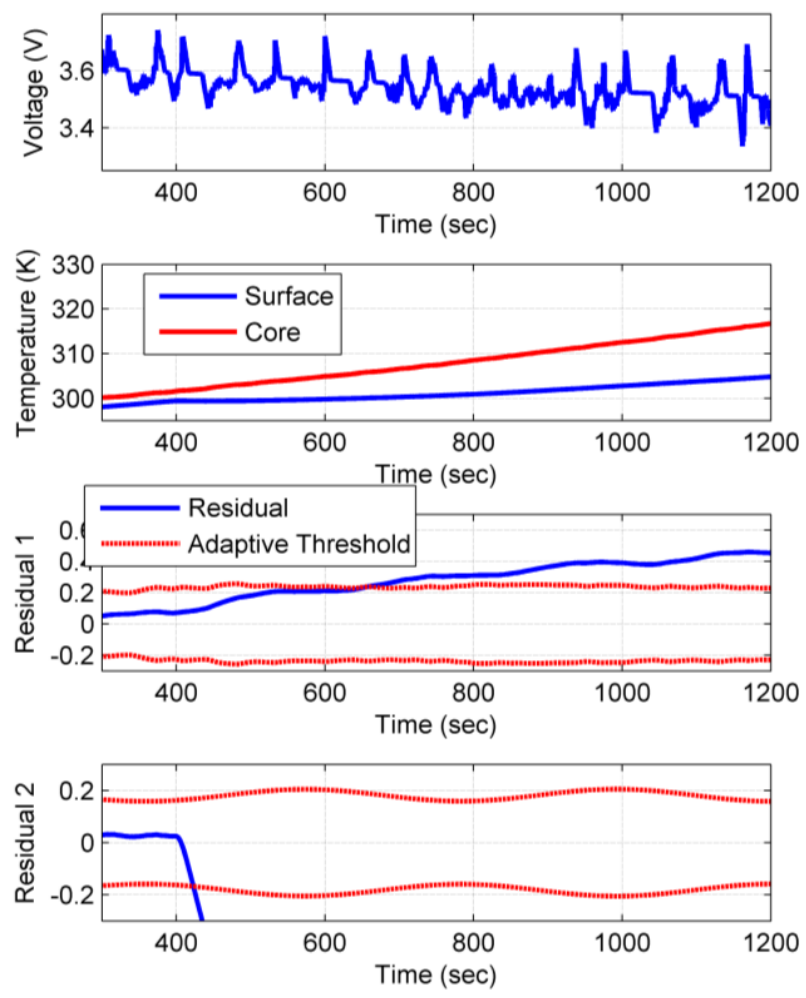

Figure 9: Residual responses under abrupt internal thermal resistance fault injected at $400 \mathrm{sec}$. The fault is a step-like fault with amplitude of $\mathbf{2} \times \mathbf{R}_{\mathbf{c}-\text { nominal }}$ 
Next, the robustness of the diagnostic scheme is evaluated under the presence of uncertainties. Two different sources of uncertainties have been considered: parametric deviations and measurement noise. To illustrate the performance of the scheme, Case 3 has been chosen where a step-like abrupt additive heat generation fault is injected at $550 \mathrm{sec}$.

Performance of the diagnostic scheme under parametric uncertainties: In this case, some plant model parameters $\left(R, Q, C_{s}\right.$ and $\left.C_{c}\right)$ are deviated from their nominal values. The residual responses are shown in Fig. 10 under some of these parametric deviations. It is noted in the simulation studies that the diagnostic scheme performs reasonably well up to $10 \%$ deviation in $R, 15 \%$ deviation in $Q, 12 \%$ deviation in $C_{c}$ and $25 \%$ deviation in $C_{s}$. After that, the performance of the diagnostic scheme degrades. One of such instances is shown in Fig. 10 under $15 \%$ deviation in $R$.

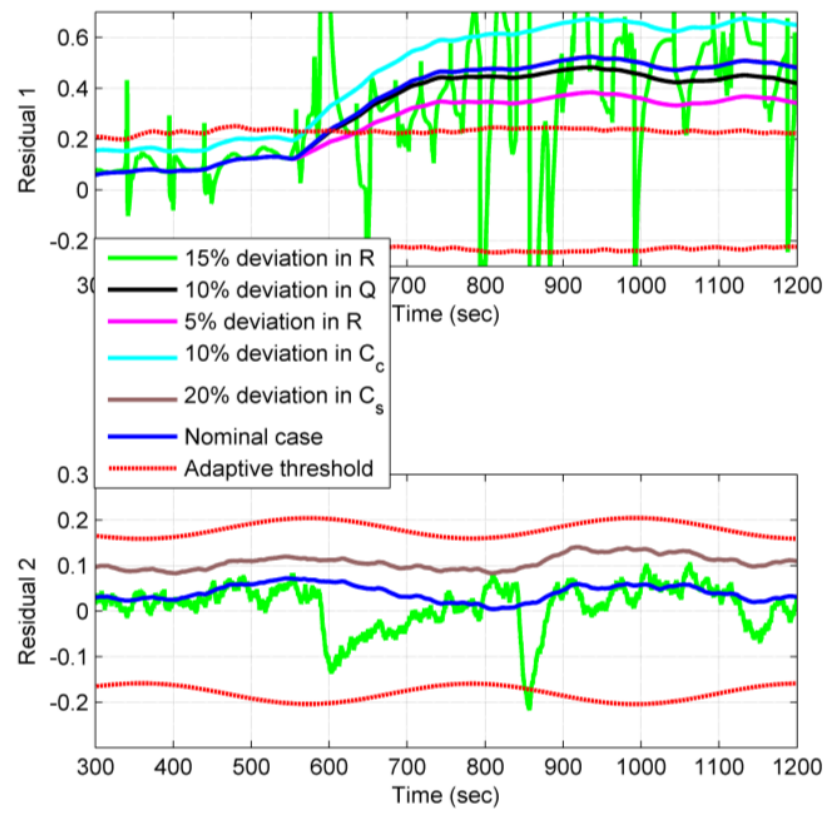

Figure 10: Residual responses under parametric deviations with abrupt additive heat generation fault injected at $550 \mathrm{sec}$.

The fault is a step-like fault with amplitude of 0.025 Watt.

Performance of the diagnostic scheme under measurement uncertainties: In this case, measurement noises are injected into the plant outputs (voltage, surface temperature and current). The residual responses are shown in Fig. 11 in presence of different levels of these measurement uncertainties. The noises are chosen as zero mean Gaussian noise with a certain standard deviation. It is noted in the simulation studies that the diagnostic scheme performs reasonably well up to $5 \mathrm{mV}, 25 \mathrm{~mA}$ and $1^{\circ} \mathrm{C}$ voltage, current and temperature measurement noise. After that, the performance of the diagnostic scheme degrades. One of such instances is shown in Fig. 11 under $50 \mathrm{~mA}$ current measurement noise.

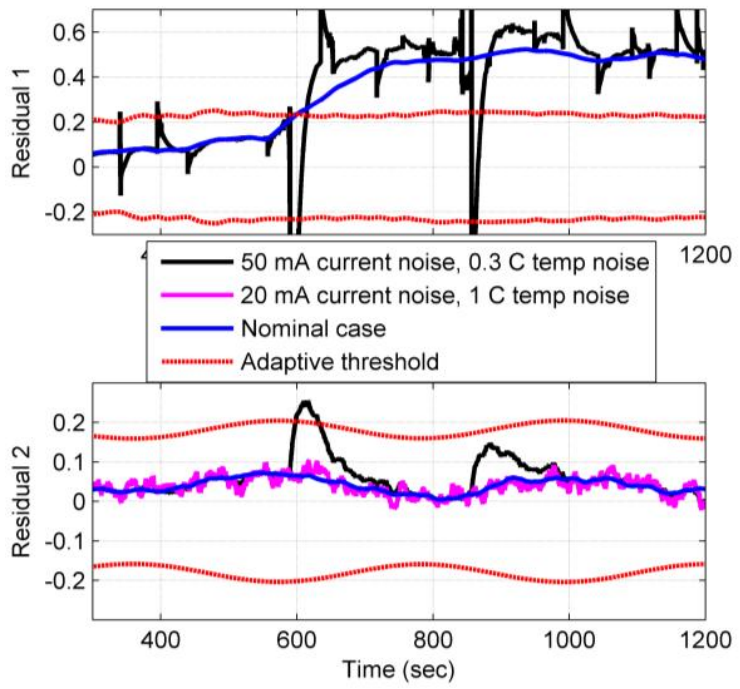

Figure 11: Residual responses under measurement noises with abrupt additive heat generation fault injected at $550 \mathrm{sec}$. The fault is a step-like fault with amplitude of 0.025 Watt.

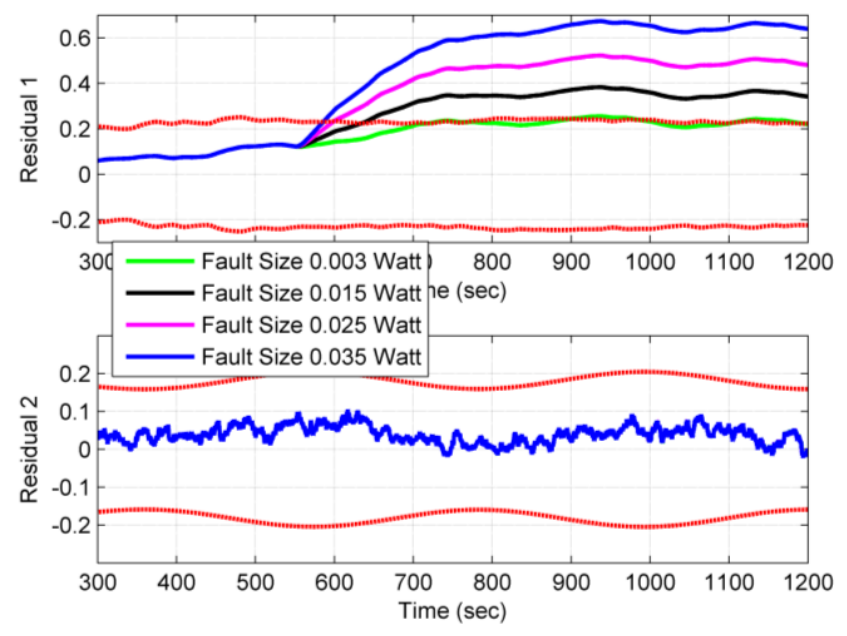

Figure 12: Residual responses under abrupt additive heat generation fault of different amplitudes, injected at $550 \mathrm{sec}$.

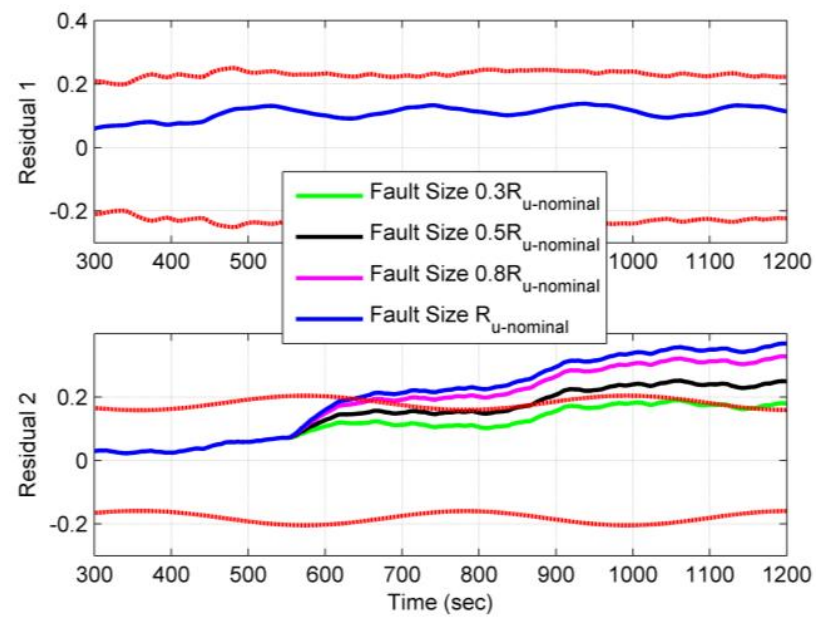

Figure 13: Residual responses under abrupt convective cooling coefficient fault of different amplitudes, injected at $550 \mathrm{sec}$. 
Minimum detectable faults and fault detection times: In this section, the fault detection times and minimum detectable faults are analysed via simulation studies. Two different types of abrupt faults are considered for this purpose: additive heat generation faults and convective cooling coefficient faults. The residual responses under additive heat generation faults with different amplitudes are shown in Fig. 12. It can be seen from Fig. 12 that the fault detection times for 0.003 Watt, 0.015 Watt, $0.025 \mathrm{Watt}$ and $0.035 \mathrm{Watt}$ faults are $168 \mathrm{sec}, 83$ $\mathrm{sec}, 40 \mathrm{sec}$ and $32 \mathrm{sec}$, respectively. As expected, the detection time becomes smaller as the fault amplitude is higher. Further, the minimum detectable fault is 0.0025 Watt. In case of thermal runaway, which is captured by the additive heat generation fault, the fault nature would be an abrupt type with high amplitude. So, the detection time for thermal runaway would be smaller. A similar study is done with respect to the convective cooling coefficient faults and the corresponding residual responses are shown in Fig. 13. It can be seen from Fig. 13 that the fault detection times for $30 \%$, $50 \%, 80 \%$ and $100 \%$ deviation of convective cooling coefficient with respect to its nominal value are $600 \mathrm{sec}, 320$ sec, $107 \mathrm{sec}$ and $87 \mathrm{sec}$, respectively. The minimum detectable fault size is $30 \%$ of the nominal value of the convective cooling coefficient.

\subsection{Experimental Studies}

An experimental study is presented here to understand the effectiveness of the diagnostic scheme under a convection coefficient fault.

Fault Injection: The convective fault has been injected by changing the exposure of the battery surface to its surroundings. This was achieved by insulating the whole of the battery surface with leather insulating material after a certain time during discharge. The current profile under consideration was $1 \mathrm{C}$ constant discharge.

Note: The injection of thermal faults essentially creates thermal imbalances in the battery and hence affects the battery health. Therefore, it is extremely important to diagnose the thermal faults, not only from a safety point of view, but also to increase battery longevity. The details of how the thermal faults/imbalances degrade the battery health, can be found in Merla et al (2016).

Offline Computation of the Uncertainty Bounds: To compute the uncertainty bounds, the following steps are used:

-The uncertainty model is chosen in the form of equations (28)-(29).

-The open-loop model error is found by subtracting the openloop model outputs (surface and core temperatures) and the corresponding measurements. The measurements were collected in the SOC range of 50-80\%, temperature range of $15-40^{\circ} \mathrm{C}$ and current range up to $2 \mathrm{C}$. Current profiles include constant currents, pulse discharge and ramp currents.

-Consider (26)-(29) as an uncertain plant model and the following as a nominal plant model (without uncertainty). $\dot{x}_{1}=a_{11} x_{1}+a_{12} x_{2}+b_{11} u_{1}^{2} f_{R}\left(S O C, x_{1}\right)$

$\dot{x}_{2}=a_{21} x_{1}+a_{22} x_{2}+b_{22} u_{2}$

The dynamics of the open-loop model error are

$$
\begin{aligned}
& \dot{\tilde{x}}_{1}=a_{11} \tilde{x}_{1}+a_{12} \tilde{x}_{2}+n_{1} \\
& \dot{\tilde{x}}_{2}=a_{21} \tilde{x}_{1}+a_{22} \tilde{x}_{2}+n_{2}
\end{aligned}
$$

From the physical properties of the system, the matrix $A=\left[\begin{array}{ll}a_{11} & a_{12} \\ a_{21} & a_{22}\end{array}\right]$ is Hurwitz. Therefore, under asymptotic condition, the error equation becomes:

$$
\left[\begin{array}{l}
n_{1} \\
n_{2}
\end{array}\right]=-A\left[\begin{array}{l}
\tilde{x}_{1} \\
\tilde{x}_{2}
\end{array}\right]
$$

Note that, $\tilde{x}_{2}$ and $\tilde{x}_{2}$ are the open-loop model errors computed in the previous step. Then, $n_{1}$ and $n_{2}$ can be calculated from (51).

-The upper bounds of $n_{1}$ and $n_{2}$ can be written as:

$$
\begin{aligned}
& \left|n_{1}\right| \leq\left|n_{1}\right| \max _{\text {max }} \\
& =k_{11-\max }\left|x_{1}\right|+k_{12-\max }\left|x_{2}\right|+k_{13-\max }\left|u_{1}\right|+k_{14-\max } \\
& \left|n_{2}\right| \leq\left.\left|n_{2}\right|\right|_{\text {max }} \\
& =k_{21-\max }\left|x_{1}\right|+k_{22-\max }\left|x_{2}\right|+k_{23-\max }\left|u_{2}\right|+k_{24-\max }
\end{aligned}
$$

-The parameters $k_{i j-\max }$ are tuned to find $\left|n_{1}\right|_{\max }$ and $\left|n_{2}\right|_{\text {max }}$ which bounds the $n_{1}$ and $n_{2}$ under all conditions.
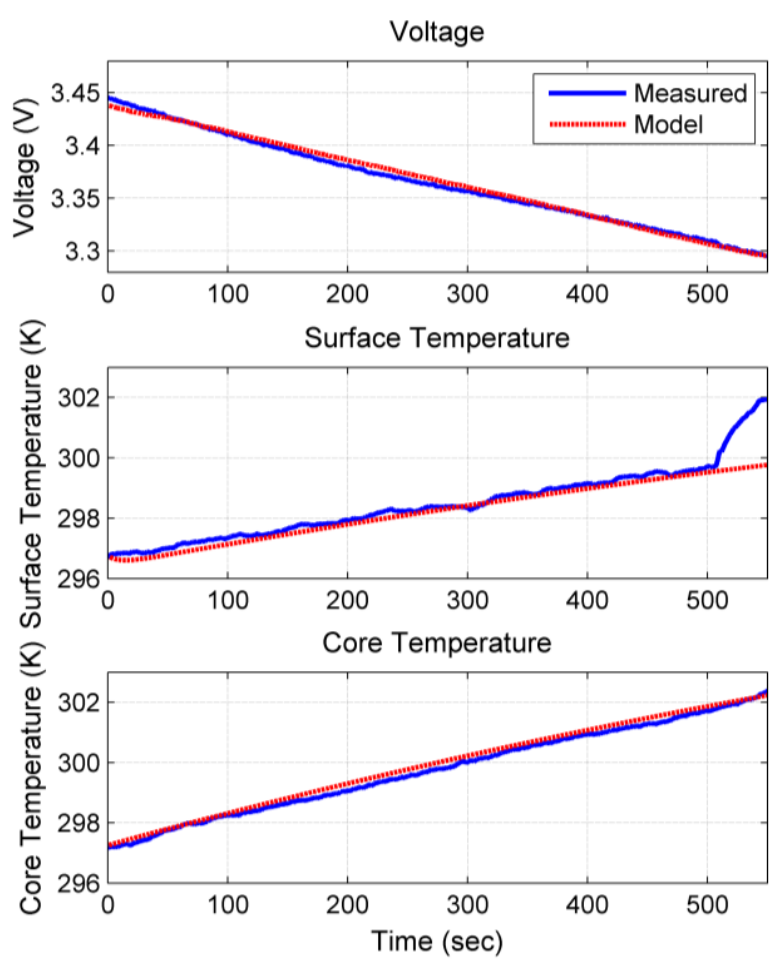

Figure 14: Comparison of model voltage and temperature responses with measured data under $1 \mathrm{C}$ discharge current. The convective fault was injected at $500 \mathrm{sec}$. 

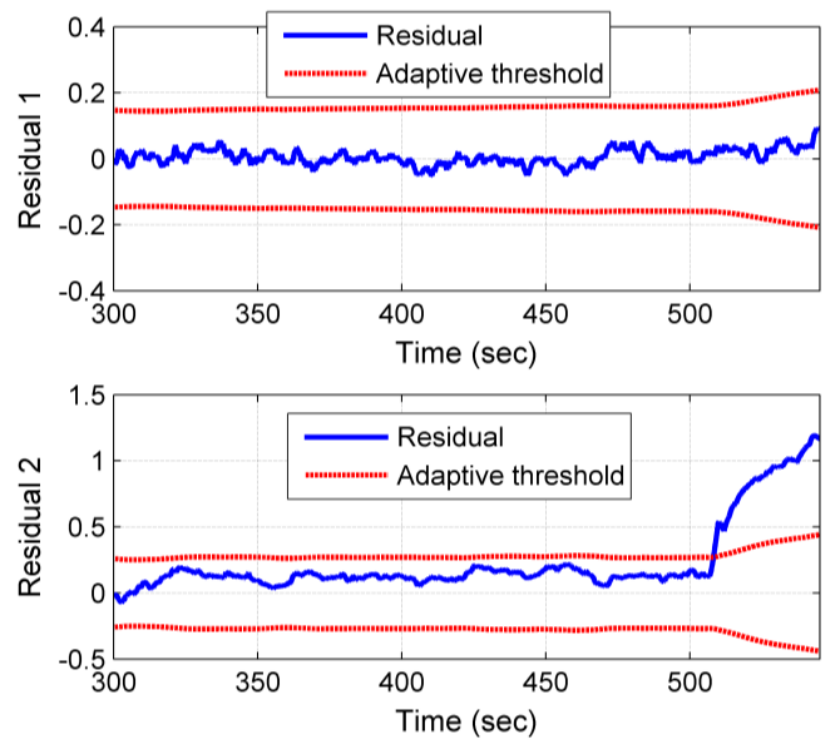

Figure 15: Residual responses under convective fault injected at $500 \mathrm{sec}$.

Open-loop Model Validation: The voltage and temperature responses from the nominal open-loop model are compared with the measured experimental data under $1 \mathrm{C}$ constant discharge and shown in Fig. 14. Note that, the convective cooling fault was injected at $500 \mathrm{sec}$ and hence the surface temperature started deviating from its nominal rate at that point. However, the core temperature and voltage remained unaffected. Therefore, there is a mismatch in surface temperature between model data and measured data after 500 sec.

Performance of the Diagnostic Scheme: Under the fault injection at $500 \mathrm{sec}$, the residual responses along with the adaptive thresholds are shown in Fig. 15. The residual responses confirm the occurrence of the convection coefficient fault as the residual $1\left(r_{1}\right)$ remains within the threshold whereas the residual $2\left(r_{2}\right)$ crosses the threshold. The fault detection time is $8 \mathrm{sec}$. Note that, the adaptive thresholds experience significant change after $500 \mathrm{sec}$. This is because the adaptive thresholds are driven by measured surface temperature which deviates significantly after the fault occurrence.

\section{Conclusion}

In this paper, a fault diagnostic scheme has been presented to diagnose thermal faults in the Lithium-ion battery cell. A two-state thermal model capturing the core and surface temperature of the battery has been considered in developing the scheme. A nonlinear observer with measured surface temperature and reconstructed core temperature output error injection has been designed using Lyapunov analysis. The output errors of the observer are treated as residual signals for detecting and isolating the faults in the system. To enhance the robustness of the scheme to modelling uncertainties, an adaptive threshold generator has been designed based on the known bounds of the uncertainties. The residuals are compared with these adaptive thresholds to decide fault occurrence. Simulation and experimental studies conducted in a commercial Lithium-ion battery cell are included to illustrate the effectiveness of the scheme.

For future extension of this research, the following can be considered: 1) development of a battery thermal diagnostic scheme based on more comprehensive thermal models, e.g. distributed parameter battery thermal models and, 2) thermal fault-tolerant control of batteries where the thermal management algorithm minimizes the effect of thermal faults on the batteries in order to reduce battery degradation.

\section{ACKNOWLEDGEMENTS}

Research supported by the US Department of Energy GATE program under grant number DE-EE0005571.

\section{REFERENCES}

Alessandri, A., Caccia, M., \& Veruggio, G. (1999). Fault detection of actuator faults in unmanned underwater vehicles. Control Engineering Practice, vol. 7, no. 3, pp. 357-368.

Bandhauer, T. M., Garimella, S., \& Fuller, T. F. (2011). A critical review of thermal issues in lithium-ion batteries. Journal of the Electrochemical Society, vol. 158, no. 3, pp. R1-R25.

Biron, Zoleikha Abdollahi, Pierluigi Pisu, and Beshah Ayalew. (2015). Observer-Based Diagnostic Scheme for Lithium-Ion Batteries. Proceedings of ASME 2015 Dynamic Systems and Control Conference, pp. V002T19A003.

Charkhgard, M., \& Farrokhi, M. (2010). State-of-charge estimation for lithium-ion batteries using neural networks and EKF. IEEE Transactions on Industrial Electronics, vol. 57, no. 12, pp. 4178-4187.

Chen, S. C., Wan, C. C., \& Wang, Y. Y. (2005). Thermal analysis of lithium-ion batteries. Journal of Power Sources, vol. 140, no. 1, pp. 111-124.

Debert, M., Colin, G., Bloch, G., \& Chamaillard, Y. (2013). An observer looks at the cell temperature in automotive battery packs. Control Engineering Practice, vol. 21, no.8, pp. 1035-1042.

Dey, S., Ayalew, B. and Pisu, P. (2015a). Nonlinear Robust Observers for State-of-Charge Estimation of Lithium-Ion Cells Based on a Reduced Electrochemical Model. IEEE Transactions on Control Systems Technology, vol.23, no.5, pp.1935-1942.

Dey, S., Ayalew, B. and Pisu, P. (2015b). Nonlinear Adaptive Observer for a Lithium-ion Battery Cell Based on Coupled Electrochemical-Thermal Model. ASME Journal of Dynamic Systems, Measurement, and Control, vol. 137, no. 11, pp. 111005.

Dey, S., Mohon, S., Pisu, P and Ayalew, B. (2015c). Sensor Fault Detection, Isolation and Estimation in Li-ion Batteries. accepted in IEEE Transactions on Control 
Systems Technology, available online, DOI 10.1109/TCST.2016.2538200.

Dey, S., and Ayalew, B. (2015d). A Diagnostic Scheme for Detection, Isolation and Estimation of Electrochemical Faults in Lithium-ion Cells. Proceedings of ASME 2015 Dynamic Systems and Control Conference, pp. V001T13A001.

Dey, S., Abdollahi, Z., Tatipamula, S., Das, N., Mohon, S., Ayalew, B., and Pisu, P. (2015e). On-board Thermal Fault Diagnosis of Lithium-ion Batteries For Hybrid Electric Vehicle Application. Proceedings of IFAC Workshop on Engine and Powertrain Control, Simulation and Modeling, pp. 389-394, 2015.

Ding, X. and Frank, P. M. (1991). Frequency domain approach and threshold selector for robust model-based fault detection and isolation. In Preprint of IFAC/IMACS Symposium SAFEPROCESS'91, vol. 1, pp. 307-312.

Doughty, D. H., Butler, P. C., Jungst, R. G., \& Roth, E. P. (2002). Lithium battery thermal models. Journal of Power Sources, vol. 110, no. 2, pp. 357-363.

Edwards, C., Spurgeon, S. K., \& Patton, R. J. (2000). Sliding mode observers for fault detection and isolation. Automatica, vol. 36, no. 4, pp. 541-553.

Emami-Naeini, A., Akhter, M., and Rock, M. (1988). Effect of model uncertainty on failure detection: the threshold selector. IEEE Trans. on Automatic Control, vol. 33, no. 2, pp. 1106-1115.

Feig, P., Billitteri, F., Longo, S., \& Auger, D. (2014). Sensors-models trade-offs in battery state estimation. In Hybrid and Electric Vehicles Conference (HEVC 2014), 5th IET, pp. 1-7.

Gao, Z., \& Wang, H. (2006). Descriptor observer approaches for multivariable systems with measurement noises and application in fault detection and diagnosis. Systems \& Control Letters, vol. 55, no. 4, pp. 304-313.

Gao, Z., Ding, S. X., \& Cecati, C. (2015). Real-time fault diagnosis and fault-tolerant control. IEEE Transactions on Industrial Electronics, vol. 62, no. 6, pp. 3752-3756.

Gertler, Janos. Fault detection and diagnosis in engineering systems. CRC press, 1998.

Gould, C. R., Bingham, C. M., Stone, D. A., \& Bentley, P. (2008). Novel battery model of an all-electric personal rapid transit vehicle to determine state-of-health through subspace parameter estimation and a Kalman estimator. Proceedings of International Symposium on Power Electronics, Electrical Drives, Automation and Motion, pp. 1217-1222.

Guo, G., Long, B., Cheng, B., Zhou, S., Xu, P., \& Cao, B. (2010). Three-dimensional thermal finite element modeling of lithium-ion battery in thermal abuse application. Journal of Power Sources, vol. 195, no. 8, 2393-2398.
Hallaj, S. A., Maleki, H., Hong, J., and Selman, J. (1999). Thermal modeling and design considerations of lithiumion batteries. Journal of Power Sources, vol. 83, pp. 1-8.

He, H., Xiong, R., Zhang, X., Sun, F., \& Fan, J. (2011). State-of-charge estimation of the lithium-ion battery using an adaptive extended Kalman filter based on an improved Thevenin model. IEEE Transactions on Vehicular Technology, vol. 60, no. 4, pp. 1461-1469.

Hu, Y., and Yurkovich, S. (2012). Battery cell state-of-charge estimation using linear parameter varying system techniques. Journal of Power Sources, vol. 198, pp. 338 350.

Kim, I.-S. (2006).The novel state of charge estimation method for lithium battery using sliding mode observer. Journal of Power Sources, vol. 163, no. 1, pp. 584-590.

Kim, G. H., Pesaran, A., \& Spotnitz, R. (2007). A threedimensional thermal abuse model for lithium-ion cells. Journal of Power Sources, 170(2), 476-489.

Kim, Y., Mohan, S., Siegel, J. B., Stefanopoulou, A. G., \& Ding, Y. (2014). The estimation of temperature distribution in cylindrical battery cells under unknown cooling conditions. IEEE Transactions on Control Systems Technology, vol. 22, no. 6, pp. 2277-2286.

Klein, R., Chaturvedi, N. A., Christensen, J., Ahmed, J., Findeisen, R., and Kojic, A. (2013). Electrochemical Model Based Observer Design for a Lithium-Ion Battery. IEEE Transactions on Control Systems Technology, vol. 21, no. 2, pp. 289-301.

Lin, X. , Perez, H. E. , Siegel, J. B. , Stefanopoulou, A. G. , Li, Y., Anderson, R.D. , Ding, ,Y., and Castanier, M. P. (2013). Online parameterization of lumped thermal dynamics in cylindrical Lithium ion batteries for core temperature estimation and health monitoring. IEEE Transaction on Control Systems Technology, vol. 21, no. 5, pp. 1745-1755, September.

Liu, Z., Ahmed, Q., Rizzoni, G., and He, H. (2014). Fault Detection and Isolation for Lithium-Ion Battery System Using Structural Analysis and Sequential Residual Generation. Proceedings of 2014 ASME Dynamic Systems and Control Conference (DSCC), pp. V002T36A005.

Lombardi, W., Zarudniev, M., Lesecq, S., and Bacquet, S. (2014). Sensors fault diagnosis for a BMS. Proceedings of 2014 European Control Conference (ECC), pp. 952957.

Maleki, H., and Shamsuri, A. K. (2003). Thermal analysis and modeling of a notebook computer battery. Journal of Power Sources, vol. 115, pp. 131-136.

Marcicki, J., Onori, S., and Rizzoni, G. (2010). Nonlinear fault detection and isolation for a lithium-ion battery management system. Proceedings of ASME 2010 Dynamic Systems and Control Conference, pp. 607-614. 
Merla, Y., Wu, B., Yufit, V., Brandon, N. P., Martinez-Botas, R. F., \& Offer, G. J. (2016). Novel application of differential thermal voltammetry as an in-depth state-ofhealth diagnosis method for lithium-ion batteries. Journal of Power Sources, vol. 307, pp. 308319.

Moura, S. J., Chaturvedi, N. A., and Krstic, M. (2012). PDE estimation techniques for advanced battery management systems - Part I: SOC estimation. Proceedings of 2012 American Control Conference (ACC), pp. 559-565.

Park C. W. and Jaura, A. K. (2003). Dynamic thermal model of li-ion battery for predictive behavior in hybrid and fuel cell vehicles. SAE transactions, vol. 112, no. 3 pp. 1835-1842.

Papazoglou, A., Longo, S., Auger, D., \& Assadian, F. (2014). Nonlinear Filtering Techniques Comparison for Battery State Estimation. Journal of Sustainable Development of Energy, Water and Environment Systems, vol. 2, no. 3, pp. 259-269.

Patton, R. J., Putra, D., \& Klinkhieo, S. (2010). Friction compensation as a fault-tolerant control problem. International Journal of Systems Science, vol. 41, no. 8, pp. 987-1001.

Patton, R. J., \& Klinkhieo, S. (2009). An adaptive approach to active fault-tolerant control. The Open Automation and Control Systems Journal, vol. 2, no. 1.

Plett, G. L. (2004). Extended Kalman filtering for battery management systems of LiPB-based HEV battery packs: Part 1. Background. Journal of Power sources, vol. 134, no. 2, pp. 252-261.

Richardson, Robert R., Peter T. Ireland, and David A. Howey (2014). Battery internal temperature estimation by combined impedance and surface temperature measurement. Journal of Power Sources, vol. 265, pp. 254-261.

Singh, A., Izadian, A., and Anwar, S. (2013). Fault diagnosis of Li-Ion batteries using multiple-model adaptive estimation. Proceedings of IECON 2013-39th Annual Conference, pp. 3524-3529.

Smith, K., and Wang, C.-Y. (2006). Power and thermal characterization of a lithium-ion battery pack for hybridelectric vehicles. Journal of Power Sources, vol. 160, p. 662-673.

Wang, Q., Ping, P., Zhao, X., Chu, G., Sun, J., \& Chen, C. (2012). Thermal runaway caused fire and explosion of lithium ion battery. Journal of power sources, vol. 208, pp. $210-224$.

Yan, X., \& Edwards, C. (2007). Nonlinear robust fault reconstruction and estimation using a sliding mode observer. Automatica, vol. 43, pp. 1605-1614.

Zhang, Xiaodong, Marios M. Polycarpou, and Thomas Parisini (2002). A robust detection and isolation scheme for abrupt and incipient faults in nonlinear systems. IEEE Transactions on Automatic Control, vol. 47, no. 4, pp. 576-593.

Zhong, M., Ding, S. X., Lam, J., \& Wang, H. (2003). An LMI approach to design robust fault detection filter for uncertain LTI systems. Automatica, vol. 39, no. 3, pp. 543-550. 